\title{
Identification of Drug Related Problems among Chronic Kidney Disease Patients in A Tertiary Care Hospital
}

\author{
${ }^{1}$ Consultant Nephrologist, Vijaya Hospital, Chennai, India \\ ${ }^{2}$ Pharm D intern, K.K College of Pharmacy, Gerugambakkam, Chennai, India \\ ${ }^{3}$ Professor, K.K College of Pharmacy, Gerugambakkam, Chennai-128, India
}

R. Sakthirajan ${ }^{1}$, Sheena Varghese ${ }^{2}$, Roshni $\mathrm{S}^{2}$, Sai Laharika $\mathrm{P}^{2}$, Muhammed Sufiyan M. J², S. Ramalakshmi ${ }^{3 *}$

\author{
DOI: $10.36348 /$ sjmps.2019.v05i11.005 \\ | Received: 29.10.2019 | Accepted: 05.11.2019 | Published: 12.11.2019 \\ *Corresponding author: Sankaralingam Ramalakshmi
}

\section{Abstract}

Medication therapy to Chronic Kidney Disease patients is complex because of the co morbidities and the major risk associated with these patients. A prospective, cross sectional study was conducted for a period of 6 months in a tertiary care hospital. A total of 130 patients, aged 18 years or older, diagnosed with all stages of Chronic Kidney Disease were included in the study. Patient data were collected from the patient medical records, the prescriptions were analyzed and the drug related problems were identified and classified according to Hepler and Strand. Descriptive analysis was done for age, gender, stages, drugs and Drug Related Problems. A total of 1454 drugs were prescribed for the patients with an average of $13.3 \pm 3.53$ drugs per prescription. An average of $1.25 \pm 1.23$ Drug Related Problems per prescription were observed among the study population. The most common Drug Related Problems identified was Adverse Drug Reactions $(11.53 \%)$ and Drug combinations to use with caution and need close monitoring $(11.53 \%)$, followed by over dose $9.23 \%$. Other Drug Related Problems identified were untreated indication 7.69\%, Drug without indication 3.08\%, improper drug selection $1.53 \%$, Treatment duplicity $0.76 \%$, and potential Drug interactions $80.00 \%$.Continual identification and resolution of Drug Related Problems in Chronic Kidney Disease could play a vital role in achieving better clinical outcomes.

Keywords: Drug Related Problems, Chronic Kidney Disease, Adverse Drug Reactions, polypharmacy, Co morbidities, Drug interactions.

Copyright @ 2019: This is an open-access article distributed under the terms of the Creative Commons Attribution license which permits unrestricted use, distribution, and reproduction in any medium for non-commercial use (NonCommercial, or CC-BY-NC) provided the original author and source are credited.

\section{INTRODUCTION}

Chronic Kidney Disease (CKD) is defined as the presence of kidney damage or decreased Glomerular Filtration Rate (GFR) for 3 months or more [1]. Medication therapy to Chronic Kidney Disease patients is complex because of the comorbidities and the major risk associated with these patients. CKD is a global threat to health in general and for developing countries in particular, because therapy is expensive and lifelong [2]. It is highly prevalent and is increasing in public health concern as the number of people affected by it is increasing each year [3]. As CKD progresses, the drugs that are given for these patients also increases and the prevalence of drug related problems also increases [4]

A Drug Related Problem is an event occurring as a result of the drug therapy that actually or potentially interferes with desired health outcomes [5]. A thorough knowledge of DRPs may help in identifying DRPs, resolving actual DRPs and preventing potential DRPs for the provision of better patient care.

\section{Categories of DRPs}

According to Hepler and Strand classification, DRPs are broadly classified into eight categories [4].

Indication without drug (IWD) / Untreated Indication

Indication without drug means the patient is not receiving a drug for a given medical condition despite the need for such a drug [6].

\section{Drug without Indication (DWI)}

Drug without indication (DWI) means the patient has no valid medical indication for taking a certain drug [6].

\section{Improper Drug Selection (IDS)}

Improper drug selection (IDS) means the patient is taking the wrong drug for a given medical condition [6]. 
Sub Therapeutic Dosage (STD) / Under Dosage (UD)

Sub-therapeutic dosage (STD) means the patient is taking too little of the correct drug for a given medical condition [6].

\section{Over Dosage (OD)}

Over-dosage (OD) means patient is taking too much of the correct drug for a given medical condition [6].

\section{Adverse Drug Reactions (ADRs)}

Adverse drug reaction (ADR) means that unwanted/unpleasant or harmful drug effects caused a medical condition in a patient $[6,7]$.

\section{Drug Interactions (DI)}

DI can be defined as the set of alterations introduced up on the therapeutic effect of a given drug stemming from the co-administration of one or more medications $[8,9]$.

\section{Failure to Receive Drug (FRD)}

Failure to receive drug (FRD) means patient is not receiving prescribed medications for a given medical condition [6].

\section{METHODOLOGY}

This study was a Prospective cross sectional, observational study conducted for a period of 6 months with a total of 130 patients whose age greater than 18 years or older, diagnosed with all stages of Chronic Kidney Disease in a tertiary care hospital. Out patients, those having hepatic dysfunction, Pregnant and lactating women and critically ill patients were excluded from the study. The study was approved by Institutional Ethics Committee (IEC -VCMR) EC/LTR/2018/010 of Vijaya Hospital. The data from the case sheets were taken in a data collection form specially designed for the study which includes the patient's demographics (age, sex, weight), co morbidities, past and present medications and medical history, fresh complaints, drug treatment regimens and lab reports $(\mathrm{CBC}, \mathrm{RFT}$, Blood Glucose levels, lipids and other tests).

\section{CALCULATION OF GFR}

Renal function i.e. GFR will be calculated using CKD - EPI calculators which involve the following equation

eGFR = $141 \times \min (\mathrm{SCr} / \mathrm{\kappa}, 1) \alpha \times \max (\mathrm{SCr} / \mathrm{\kappa}, 1)-1.209 \times 0.993 \mathrm{Age}$

$\mathrm{x} 1.018$ [if female] $\mathrm{x} 1.159$ [if Black]

\section{PRESCRIPTION ANALYSIS}

The patient's case notes and drug chart were analyzed for the DRPs:

- Dosage Adjustments according to KDIGO Guidelines.

- Drug Interactions were assessed through MICROMEDEX Software.

- ADRs were assessed through Naranjo Algorithm were classified into definite (912), probable (5-8), possible (1-4), or doubtful (0).

\section{DRP DOCUMENTATION}

The identified DRPs were documented in DRP documentation form and necessary interventions were reported.

\section{FOLLOW UP}

Daily follow up until discharge were carried out.

\section{STATISTICAL ANALYSIS}

All the data collected were tabulated and analyzed statistically using STATA version 11.0 Descriptive analysis was done for age, gender, stages of CKD drugs prescribed and DRPs. Continuous variables was represented as Mean (SD) and categorical variables was represented as Frequency (percentage). Spearman's
Correlation was performed to determine the occurrence of DRPs correlation with age, co morbidities and stages. The $p$ value of $<0.05$ was considered as statistically significant.

\section{RESULTS}

A total of 130 patients were selected as per inclusion and exclusion criteria. Their demographic details were collected which is represented in the Table1.

Table-1: Demography of the Study Population

\begin{tabular}{|c|c|c|}
\hline \multirow{2}{*}{ Age in years Range } & No. of Patients ( n=130) \\
\cline { 2 - 3 } & Frequency & Percentage \\
\hline $18-40$ & 3 & $2.30 \%$ \\
\hline $41-60$ & 57 & $43.84 \%$ \\
\hline $\mathbf{6 1 - 8 0}$ & $\mathbf{6 2}$ & $\mathbf{4 7 . 6 9 \%}$ \\
\hline$>80$ & 8 & $6.15 \%$ \\
\hline Gender \\
\hline Female Comorbidities \\
\hline \multicolumn{3}{|c|}{118} \\
\hline Hypertension & 89 & $27.98 \%$ \\
\hline Diabetes Mellitus & 64 & $20.12 \%$ \\
\hline Coronary Artery Disease & 29 & $9.11 \%$ \\
\hline Others Dialysis \\
\hline \multicolumn{3}{|c|}{$65.38 \%$} \\
\hline Yes & 65 & $50.0 \%$ \\
\hline No & 65 & $50.0 \%$ \\
\hline
\end{tabular}

PREVALENCE OF CKD 
The prevalence of Chronic Kidney Disease in the study population according to the stages of CKD as per National Kidney Disease Foundation classification was as follows: none of the patients were in CKD-I stage, $1(0.76 \%)$ were in CKD-II, $28(21.53 \%)$ were in CKD-III, 36(27.69\%) were in CKD-IV, and 65(50.0\%) were in CKD-V stage.

\section{DRP-Drug Related Problems}

Drugs prescribed in the study population is represented in Table-2. A total of 163 DRPs were identified which is indicated in Table-3. ADR and Contraindication were predominantly identified DRP.

Table-2: Drugs Prescribed In CKD Patients

\begin{tabular}{|c|c|c|c|}
\hline Drugs & Category & Frequency & Percentage \\
\hline \multirow{9}{*}{ Antihypertensives \& Diuretics $(\mathrm{n}=310)$} & Calcium channel blockers & 70 & $38.04 \%$ \\
\hline & Centrally acting drugs & 45 & $24.45 \%$ \\
\hline & Beta blockers & 35 & $19.02 \%$ \\
\hline & Alpha blockers & 20 & $10.86 \%$ \\
\hline & Angiotensin receptor blockers & 13 & $7.06 \%$ \\
\hline & Angiotensin converting enzyme inhibitors & 1 & $0.54 \%$ \\
\hline & Furosemide/Torsemide & 87 & $28.43 \%$ \\
\hline & Spironolactone & 18 & $5.88 \%$ \\
\hline & Metalazone & 11 & $3.59 \%$ \\
\hline \multirow{3}{*}{$\begin{array}{l}\text { Anti-Diabetics } \\
(\mathrm{n}=54)\end{array}$} & Metformin & 5 & $9.25 \%$ \\
\hline & $\begin{array}{l}\text { Newer drugs } \\
\text { (Saxagliptin,Linagliptin,Vildagliptin) }\end{array}$ & 4 & $7.4 \%$ \\
\hline & Insulin & 45 & $83.3 \%$ \\
\hline \multirow[t]{5}{*}{ Phosphate Binders and Iron Supplements $(\mathrm{n}=62)$} & Sevelamer & 13 & $10.0 \%$ \\
\hline & Erythropoetin & 30 & $48.83 \%$ \\
\hline & Folic acid & 9 & $14.51 \%$ \\
\hline & Ferrous fumarate + Folic acid & 4 & $6.45 \%$ \\
\hline & Ferrous sulphate + Folic acid & 3 & $4.83 \%$ \\
\hline \multirow[t]{4}{*}{ Calcium and Vitamin D Supplements $(n=46)$} & Calcium carbonate + Vitamin D3 & 18 & $39.13 \%$ \\
\hline & Cholecalciferol & 15 & $32.60 \%$ \\
\hline & Calcitriol & 12 & $26.08 \%$ \\
\hline & Calcium citrate + Magnesium + Vitamin D3 & 1 & $2.17 \%$ \\
\hline \multirow[t]{12}{*}{ Antibiotics $(\mathrm{n}=175)$} & Cefoperazone+ Sulbactam & 37 & $21.14 \%$ \\
\hline & Piperacillin+Tazobactum & 23 & $13.14 \%$ \\
\hline & Meropenem & 18 & $10.28 \%$ \\
\hline & Ciprofloxacin & 17 & $9.71 \%$ \\
\hline & Clindamycin & 15 & $8.57 \%$ \\
\hline & Linezolid & 14 & $8.0 \%$ \\
\hline & Ceftriaxone & 12 & $6.85 \%$ \\
\hline & Metronidazole & 10 & $5.71 \%$ \\
\hline & Amikacin & 7 & $4.0 \%$ \\
\hline & Azithromycin & 6 & $3.42 \%$ \\
\hline & Vancomycin & 6 & $3.42 \%$ \\
\hline & Doxycycline & 5 & $2.85 \%$ \\
\hline \multirow[t]{19}{*}{ Other Drugs $(\mathrm{n}=807)$} & Antianginal & 129 & $13.9 \%$ \\
\hline & $\mathrm{PPI} / \mathrm{H}_{2}$ Receptor Antagonist & 127 & $13.75 \%$ \\
\hline & Antiplatelet & 99 & $10.72 \%$ \\
\hline & Statins & 75 & $8.1 \%$ \\
\hline & Analgesics & 52 & $5.63 \%$ \\
\hline & Anticonvulsant & 47 & $5.09 \%$ \\
\hline & Sodium bicarbonate & 45 & $4.87 \%$ \\
\hline & Antiemetics & 42 & $4.5 \%$ \\
\hline & Thyroid drugs & 30 & $3.25 \%$ \\
\hline & Magnesium and Potassium Supplements & 24 & $2.60 \%$ \\
\hline & Anticoagulant & 23 & $2.49 \%$ \\
\hline & Febuxostat & 23 & $2.49 \%$ \\
\hline & Corticosteroids & 12 & $1.30 \%$ \\
\hline & Antifungal & 12 & $1.30 \%$ \\
\hline & Acetylcysteine & 12 & $1.30 \%$ \\
\hline & Dobutamine & 10 & $1.08 \%$ \\
\hline & Silodosin & 8 & $0.86 \%$ \\
\hline & Digoxin & 5 & $0.54 \%$ \\
\hline & Montelukast + Levocetrizine & 5 & $0.54 \%$ \\
\hline
\end{tabular}


Table-3: DRPs Identified in the Study Population

\begin{tabular}{|c|c|c|}
\hline Identified DRP & Frequency(n=130) & Percentage \\
\hline Untreated indication & 10 & $7.69 \%$ \\
\hline $\begin{array}{c}\text { Improper drug selection } \\
\text { Drug choice problem }\end{array}$ & 2 & $1.53 \%$ \\
\hline Treatment duplicity & 1 & $0.76 \%$ \\
\hline Drug combinations to use with caution and need close monitoring & 15 & $11.53 \%$ \\
\hline $\begin{array}{c}\text { Sub Therapeutic Dosage/ } \\
\text { Under Dose }\end{array}$ & 0 & 0 \\
\hline Failure To Receive Therapy & 0 & 0 \\
\hline Over dose & $\mathbf{1 2}$ & $\mathbf{9 . 2 3 \%}$ \\
\hline Adverse Reactions & $\mathbf{1 5}$ & $\mathbf{1 1 . 5 3 \%}$ \\
\hline Potential Drug Interactions & $\mathbf{1 0 4}$ & $\mathbf{8 0 . 0 \%}$ \\
\hline Drug Use without indication & 4 & $3.08 \%$ \\
\hline
\end{tabular}

Potential drug interactions found in the study population is represented in Table-4.

Table-4: Potential Drug Interactions

\begin{tabular}{|c|c|c|c|c|c|c|}
\hline Drugs & $\begin{array}{l}\text { Interacting } \\
\text { drugs }\end{array}$ & Mechanism & Effect & $\begin{array}{l}\text { Frequency } \\
(n=104)\end{array}$ & Percentage & $\begin{array}{l}\text { Monitoring } \\
\text { parameters }\end{array}$ \\
\hline \multirow{4}{*}{ Digoxin } & Nifedipine & $\begin{array}{l}\text { Additive effects on } \mathrm{AV} \\
\text { node conduction }\end{array}$ & \multirow{4}{*}{$\begin{array}{l}\text { May result in digoxin } \\
\text { toxicity; increased risk } \\
\text { of complete heart } \\
\text { block. }\end{array}$} & 3 & $2.88 \%$ & $\begin{array}{l}\text { Digoxin plasma } \\
\text { concentration }\end{array}$ \\
\hline & Metolazone & $\begin{array}{l}\text { Diuretic-induced } \\
\text { hypokalemia and } \\
\text { hypomagnesemia } \\
\text { enhance Na-K-atpase } \\
\text { inhibition by cardiac } \\
\text { glycosides }\end{array}$ & & 3 & $2.88 \%$ & $\begin{array}{lr}\text { Monitored for } \\
\text { ECG signs of } \\
\text { potassium } \\
\text { depletion }\end{array}$ \\
\hline & Spironolactone & $\begin{array}{l}\text { Inhibition of active } \\
\text { tubular secretion of } \\
\text { digoxin }\end{array}$ & & 4 & $3.84 \%$ & $\begin{array}{l}\text { Digoxin serum } \\
\text { concentration }\end{array}$ \\
\hline & Amiodarone & $\begin{array}{lr}\begin{array}{l}\text { Inhibition of } \\
\text { glycoprotein }\end{array} & \begin{array}{r}\text { by } \\
\text { amiodarone, } \\
\text { reduction of } \\
\text { clearance }\end{array} \\
\end{array}$ & & 4 & $3.94 \%$ & $\begin{array}{l}\text { Digoxin serum } \\
\text { concentration }\end{array}$ \\
\hline \multirow[t]{3}{*}{ Tramadol } & $\begin{array}{l}\text { Ranitidine/ } \\
\text { Ciprofloxacin }\end{array}$ & $\begin{array}{l}\text { Inhibition of CYP3A4- } \\
\text { mediated } \\
\text { metabolism }\end{array}$ & $\begin{array}{lr}\text { Increased } & \text { tramadol } \\
\text { exposure and increased } \\
\text { risk of respiratory } \\
\text { depression. } \\
\end{array}$ & 2 & $1.92 \%$ & $\begin{array}{l}\text { Closely monitor } \\
\text { for seizures, } \\
\text { serotonin } \\
\text { syndrome } \\
\end{array}$ \\
\hline & Alprazolam & $\begin{array}{ll}\text { Additive } & \text { CNS } \\
\text { depression } & \end{array}$ & $\begin{array}{lcr}\text { Increased } & \text { risk } & \text { of } \\
\text { respiratory } & \text { and } & \text { CNS } \\
\text { depression } & & \end{array}$ & 2 & $1.92 \%$ & $\begin{array}{l}\text { Closely monitor } \\
\text { for sedation and } \\
\text { respiratory } \\
\text { depression }\end{array}$ \\
\hline & Fluconazole & $\begin{array}{l}\text { Inhibition of } \begin{array}{l}\text { CYP3A4- } \\
\text { mediated } \\
\text { metabolism }\end{array} \\
\text { tramadol } \\
\end{array}$ & $\begin{array}{lr}\text { Increased } & \text { tramadol } \\
\text { exposure and increased } \\
\text { risk of respiratory } \\
\text { depression }\end{array}$ & 2 & $1.92 \%$ & $\begin{array}{l}\text { Monitor for } \\
\text { signs of opioid } \\
\text { withdrawal }\end{array}$ \\
\hline Metronidazole & $\begin{array}{l}\text { Ciprofloxacin/ } \\
\text { Ondansetron / } \\
\text { Fluconazole }\end{array}$ & $\begin{array}{l}\text { Additive QT-interval } \\
\text { prolongation }\end{array}$ & $\begin{array}{l}\text { Increased risk of QT- } \\
\text { interval prolongation } \\
\text { and arrhythmias }\end{array}$ & 7 & $6.73 \%$ & $\begin{array}{ll}\text { Require } & \text { ECG } \\
\text { monitoring } & \end{array}$ \\
\hline \multirow[t]{2}{*}{ Fluconazole } & Levofloxacin & $\begin{array}{l}\text { Additive effects on QT } \\
\text { interval }\end{array}$ & $\begin{array}{l}\text { Increased risk of QT } \\
\text { interval prolongation }\end{array}$ & 8 & $7.69 \%$ & $\begin{array}{ll}\begin{array}{l}\text { Require } \\
\text { monitoring }\end{array} & \\
\end{array}$ \\
\hline & Ranolazine & $\begin{array}{l}\text { Inhibition of CYP3A- } \\
\text { mediated ranolazine } \\
\text { metabolism by } \\
\text { fluconazole }\end{array}$ & $\begin{array}{l}\text { Increased ranolazine } \\
\text { plasma concentrations } \\
\text { and increased risk for } \\
\text { QT interval } \\
\text { prolongation. }\end{array}$ & 8 & $7.69 \%$ & $\begin{array}{l}\text { Require ECG } \\
\text { monitoring }\end{array}$ \\
\hline Clopidogrel & $\begin{array}{l}\text { Cilostazol /. } \\
\text { escitalopram }\end{array}$ & Additive effects & $\begin{array}{l}\text { Result in an increased } \\
\text { risk of bleeding. }\end{array}$ & 4 & $3.84 \%$ & $\begin{array}{l}\text { Close monitoring } \\
\text { for bleeding }\end{array}$ \\
\hline Acenocoumarol & fluconazole & $\begin{array}{l}\text { Decreased } \\
\text { acenocoumarol } \\
\text { metabolism }\end{array}$ & $\begin{array}{l}\text { Increased risk of } \\
\text { bleeding. }\end{array}$ & 1 & $0.96 \%$ & $\begin{array}{l}\text { Monitoring of the } \\
\text { prothrombin time }\end{array}$ \\
\hline Heparin & cefoperazone & $\begin{array}{l}\text { Inhibition of platelet } \\
\text { function, }\end{array}$ & $\begin{array}{l}\text { Increased risk of } \\
\text { bleeding }\end{array}$ & 2 & $1.96 \%$ & $\begin{array}{l}\text { Monitoring of the } \\
\text { prothrombin time }\end{array}$ \\
\hline
\end{tabular}


R. Sakthirajan et al; Saudi J Med Pharm Sci, Nov. 2019; 5(11): 949-955

\begin{tabular}{|c|c|c|c|c|c|c|}
\hline & & $\begin{array}{l}\text { synthesis of clotting } \\
\text { factors }\end{array}$ & & & & \\
\hline Zolpidem & $\begin{array}{l}\text { Alprazolam/ } \\
\text { clonazepam }\end{array}$ & Additive effects & $\begin{array}{l}\text { Increase in CNS } \\
\text { depressant effects. }\end{array}$ & 2 & $1.96 \%$ & $\begin{array}{ll}\begin{array}{l}\text { Monitor } \\
\text { sedation }\end{array} & \text { for } \\
\end{array}$ \\
\hline Clopidogrel & $\begin{array}{l}\text { Amlodipine } / \\
\text { Nifedipine }\end{array}$ & $\begin{array}{l}\text { Inhibition of CYP3A4- } \\
\text { mediated clopidogrel } \\
\text { activation }\end{array}$ & $\begin{array}{l}\text { Decreased antiplatelet } \\
\text { effect and increased } \\
\text { risk of thrombotic } \\
\text { events. }\end{array}$ & 5 & $4.80 \%$ & $\begin{array}{lr}\text { Monitored } & \text { for } \\
\text { loss } & \text { of } \\
\text { clopidogrel } & \\
\text { efficacy. } & \\
\end{array}$ \\
\hline Clonidine & $\begin{array}{l}\text { Metoprolol/ } \\
\text { Nebivolol/ } \\
\text { Bisoprolol }\end{array}$ & $\begin{array}{ll}\text { Unknown; } & \text { unopposed } \\
\text { alpha } & \text { adrenergic } \\
\text { stimulation } & \end{array}$ & $\begin{array}{l}\text { Increased risk of sinus } \\
\text { bradycardia; } \\
\text { exaggerated clonidine } \\
\text { withdrawal response }\end{array}$ & 13 & $12.5 \%$ & $\begin{array}{l}\text { Monitored } \\
\text { carefully for } \\
\text { hypertension. }\end{array}$ \\
\hline Nifedipine & voriconazole & $\begin{array}{l}\text { Inhibition of CYP3A4- } \\
\text { mediated } \\
\text { metabolism }\end{array}$ & $\begin{array}{l}\text { Increased risk of } \\
\text { hypotension, } \\
\text { bradycardia, or acute } \\
\text { renal injury. }\end{array}$ & 2 & $1.92 \%$ & $\begin{array}{l}\text { Monitoring of } \\
\text { heart rate and } \\
\text { blood pressure }\end{array}$ \\
\hline Fluconazole & Torsemide & $\begin{array}{lr}\text { Inhibition of } & \text { CYP2C9- } \\
\text { mediated } & \text { torsemide } \\
\text { metabolism } & \text { by } \\
\text { fluconazole } & \\
\end{array}$ & $\begin{array}{l}\text { Increased torsemide } \\
\text { exposure and increased } \\
\text { risk of toxicity. }\end{array}$ & 1 & $0.96 \%$ & $\begin{array}{l}\text { Monitor patients } \\
\text { for torsemide } \\
\text { toxicity }\end{array}$ \\
\hline Clopidogrel & Fluconazole & $\begin{array}{l}\text { Inhibition of CYP2C19- } \\
\text { mediated clopidogrel }\end{array}$ & $\begin{array}{l}\text { Reduced platelet } \\
\text { inhibition. }\end{array}$ & 4 & $3.84 \%$ & $\begin{array}{lr}\text { Monitored } & \text { for } \\
\text { loss } & \text { of } \\
\text { clopidogrel } & \\
\text { efficacy } & \\
\end{array}$ \\
\hline Heparin & $\begin{array}{l}\text { Glyceryl } \\
\text { trinitrate }\end{array}$ & Unknown & $\begin{array}{l}\text { Decrease in partial } \\
\text { thromboplastin time. }\end{array}$ & 2 & $1.92 \%$ & Monitor PTT \\
\hline Atorvastatin & Diltiazem & $\begin{array}{l}\text { Inhibition by diltiazem } \\
\text { of cytochrome P450 } \\
\text { 3A4-mediated } \\
\text { atorvastatin metabolism }\end{array}$ & $\begin{array}{l}\text { Increased risk } \\
\text { rhabdomyolysis. }\end{array}$ & 4 & $3.84 \%$ & $\begin{array}{c}\text { Monitor creatine } \\
\text { kinase }(\mathrm{CK}) \\
\text { levels }\end{array}$ \\
\hline Phenytoin & Quetiapine & $\begin{array}{lr}\text { Induction of CYP3A4- } \\
\text { mediated } \\
\text { metabolism }\end{array}$ & $\begin{array}{l}\text { Decreased quetiapine } \\
\text { exposure. }\end{array}$ & 2 & $1.92 \%$ & $\begin{array}{l}\text { Monitor plasma } \\
\text { concentration of } \\
\text { phenytoin }\end{array}$ \\
\hline Aspirin & $\begin{array}{l}\text { Furosemide/ } \\
\text { Spironolactone }\end{array}$ & $\begin{array}{l}\text { Decreased renal } \\
\text { prostaglandin synthesis }\end{array}$ & $\begin{array}{lr}\text { Reduced } & \text { diuretic } \\
\text { effectiveness } & \text { and } \\
\text { possible } & \\
\text { nephrotoxicity. } & \end{array}$ & 6 & $5.76 \%$ & $\begin{array}{lr}\text { Monitor } & \text { for } \\
\text { worsening renal } \\
\text { function and } \\
\text { serum potassium } \\
\text { levels }\end{array}$ \\
\hline Nifedipine & Phenytoin & $\begin{array}{l}\text { Induction of CYP3A4- } \\
\text { mediated } \\
\text { metabolism }\end{array}$ & $\begin{array}{l}\text { Decreased Nifedipine } \\
\text { exposure. }\end{array}$ & 2 & $1.92 \%$ & $\begin{array}{l}\text { Monitor plasma } \\
\text { concentration of } \\
\text { phenytoin }\end{array}$ \\
\hline Ramipril & Spironolactone & $\begin{array}{lr}\text { Increased } & \text { potassium } \\
\text { retention } & \text { secondary to } \\
\text { lowered } & \text { aldosterone } \\
\text { levels } & \\
\end{array}$ & Result in hyperkalemia & 2 & $1.92 \%$ & $\begin{array}{l}\text { Monitor } \\
\text { potasium level }\end{array}$ \\
\hline Diltiazem & Propranolol & $\begin{array}{l}\text { Additive cardiovascular } \\
\text { effects, decreased } \\
\text { metabolism of beta } \\
\text { blockers }\end{array}$ & 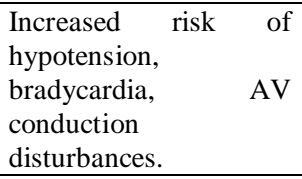 & 1 & $0.96 \%$ & $\begin{array}{l}\text { Monitor BP and } \\
\text { HR }\end{array}$ \\
\hline Aspirin & Metoprolol & $\begin{array}{l}\text { Decreased production of } \\
\text { renal prostaglandin }\end{array}$ & $\begin{array}{l}\text { Increased blood } \\
\text { pressure. }\end{array}$ & 2 & $1.92 \%$ & Monitor BP \\
\hline Amiodarone & Atorvastatin & $\begin{array}{l}\text { Inhibition of CYP3A4- } \\
\text { mediated atorvastatin } \\
\text { metabolism }\end{array}$ & $\begin{array}{l}\text { Increased risk of } \\
\text { myopathy or } \\
\text { rhabdomyolysis. }\end{array}$ & 2 & $1.92 \%$ & $\begin{array}{lr}\text { Monitor } & \text { creatine } \\
\text { kinase } & (\mathrm{CK}) \\
\text { levels } & \end{array}$ \\
\hline Ciprofloxacin & Zolpidem & Unknown & $\begin{array}{l}\text { Increased } \begin{array}{l}\text { zolpidem } \\
\text { plasma concentrations. }\end{array}\end{array}$ & 2 & $1.92 \%$ & - \\
\hline Amiodarone & Verapamil & $\begin{array}{l}\text { Inhibition of CYP3A4- } \\
\text { mediated metabolism of } \\
\text { amiodarone }\end{array}$ & 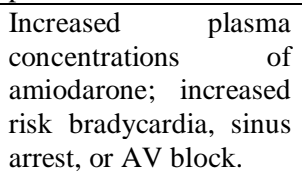 & 2 & $1.92 \%$ & Monitor HR \\
\hline
\end{tabular}

\section{Factors Contributing to DRPs}

A statistically significant difference $(\mathrm{p}=0.0232)$ between the age and DRP was observed.
The average number of drugs prescribed was predominant in the age $>60$ years. A statistically significant difference $(\mathrm{p}=0.043)$ between the $\mathrm{co}-$ 
morbities and DRPs was also observed. There was no statistically significant difference $(\mathrm{p}=0.240)$ between the stages of CKD with DRPs observed.

\section{DISCUSSION AND CONCLUSION}

It has been known that drugs may directly cause or contribute to hospital admission when the numbers of drugs are increased. DRPs can occur throughout the entire medication process and represent risk factors for ADRs and events [10]. Proper dosing can also have an economic impact on the health system. Dosage adjustment can result in avoidance of costs associated with drug related toxicity and in cost savings in terms of drug cost [11].

Majority of patients in our study population belonged to the age range 61-80 years, with highest number of DRPs $(n=90)$ which was statistically significant. A study conducted by Lesley A .Stevens et al., [3], have also reported that chronic kidney disease was a substantial concern in the elderly population with an increasing incidence of treated kidney failure resulting in dialysis.

In this study, several co-morbidities were found and the major co-morbid conditions included Hypertension (37.10\%), Diabetes mellitus (27.98\%), Coronary Artery Disease (20.12\%). This was concordant to the findings of the studies conducted by Lesley A. Stevens et al., Dena E. Rifkui et al., [12], in which they have reported that the high prevalence of CKD increased with obesity, Diabetes Mellitus , Cardiovascular drugs, Hypertension. As the age processes, co-morbid conditions increase and as a result number of prescribed drugs increase, eventually leading to increase in DRPs.

Polypharmacy can be considered as important factor for causing DRPs. In the present study, the average number of drugs per prescription was found to be $13.3 \pm 3.53$. A total of 163 DRPs were found with an average of $1.25 \pm 1.23$ DRPs per prescription and the number of DRPs were found to be increasing with an increase in number of drugs per prescription. This is in accordance with the reports of the study conducted by Katie E Cardone et al., [13], which stated that CKD patients were prescribed an average of 12 medications and were at higher risk of DRPs.

In the present study, among the identified DRPs were Adverse drug reactions and Drug combinations to use with caution and need close monitoring $(11.53 \%)$ were predominant, followed by overdose $12(9.23 \%)$. This was in accordance with the reports of a study conducted by Leape et al., [14], which reported ADEs was the most common cause. Increasing the knowledge and understanding of ADEs may help to prevent higher proportion of ADEs However in a study conducted by Manley et al. reported the major cause of DRP in CKD was medication dosing errors [15]. The proper dosing of medications for patients with renal impairment can maximize therapeutic efficacy and minimizes toxicity. In this study 15 ADRs, out of which 3 were probable and 12 were possible with respect to Naranjo Causality Assessment Scale. The ADRs observed were Clopidogrel \& Heparin induced thrombocytopenia, Prazosin \& (Isosorbide dinitrate+ Hydralazine) induced hypotension, Furosemide \& Torsemide induced hypokalemia and hyponatremia respectively. There were relative contraindications found in this study includes Isosorbide mononitrate with Sildenafil, Fluconazole with Ivabradine/ Donepezil/ Quetiapine/ Amiodarone/ Ondansetron, Linezolid with Escitalopram, Itraconazole with Ivabradine respectively.

The other DRPs identified in our study were untreated indication $10(7.69 \%)$ and Drug without indication $4(3.08 \%)$. However a study conducted by Angeles Pardo Ropez et al., [16] found 34.5\% patients require additional treatment and $14.3 \%$ had a DRP of unnecessary treatment. In the present study, untreated indications found such as decreased $\mathrm{Hb}$ levels, increased uric acid levels and potassium levels and chronic H.pylori, gastritis.

The potential Drug interactions $(80.0 \%)$ which means that there is a possibility of interactions but were not observed in these patients. Drug-Drug interactions can lead to decrease or increase in a drug effect; eventually causing sub-therapeutic or supra- therapeutic effect. These drug interactions should be closely monitored. To prevent these interaction the pharmacokinetic properties of each drug can be checked and if the half-lives of the drugs are not crossed over, they can be administered at different timings.

DRPs are common in patients with renal insufficiency maintained on haemodialysis [1]. In our study included 65 haemodialysis patients with 72 DRPs. Such patients are at higher risk, as they require complex therapeutic regimens with 5 or more medication doses per day that require frequent monitoring and dosage adjustment; they usually have other concurrent diseases including Diabetes Mellitus, Hypertension, Coronary Artery Disease and Infections.

Our study concludes that a direct proportionality was seen with number of drugs prescribed and DRPs. As observed with the increasing age and as such increase in comorbidities.

Monitoring the drug therapy for the occurrence of DRPs is a valuable way of preventing DRPs. This can be identified and reported to the physician to act and adjust drug regimens before adverse events arise and treatment failure occurs. Recognition and resolution 
of DRPs will decrease drug related morbidity and mortality.

\section{ACKNOWLEDGEMENT}

Our sincere thanks to Tamilnadu Pharmaceutical Science and Welfare Trust for providing scholarship.

\section{REFERENCES}

1. Manley, H. J., Garvin, C. G., Drayer, D. K., Reid, G. M., Bender, W. L., Neufeld, T. K.,Hebbar,S.,\& Muther, R. S. (2004). Medication prescribing patterns in ambulatory haemodialysis patients: comparisons of USRDS to a large not-for-profit dialysis provider. Nephrology Dialysis Transplantation, 19(7), 1842-1848.

2 Varughese, S., John, G. T., Alexander, S., Deborah, M. N., Nithya, N., Ahamed, I., Tamilarasi, V., \& Jacob, C. K. (2007). Pre-tertiary hospital care of patients with chronic kidney disease in India. Indian Journal of Medical Research, 126(1), 2833.

3. Coresh, J., Selvin, E., Stevens, L. A., Manzi, J., Kusek, J. W., Eggers, P., Van Lente ,F.,\& Levey, A. S. (2007). Prevalence of chronic kidney disease in the United States. Jama, 298(17), 2038-2047.

4. Patel, H. R., Pruchnicki, M. C., \& Hall, L. E. (2005). Assessment for chronic kidney disease service in high-risk patients at community health clinics. Annals of Pharmacotherapy, 39(1), 22-27.

5. Strand, L. M., Morley, P. C., Cipolle, R. J., Ramsey, R., \& Lamsam, G. D. (1990). Drugrelated problems: their structure and function. Dicp, 24(11), 1093-1097.

6. Hepler, C. D., \& Strand, L. M. (1990). Opportunities and responsibilities in pharmaceutical care. American journal of hospital pharmacy, 47(3), 533-543.

7. Pai, A. B., Cardone, K. E., Manley, H. J., Peter, W. L. S., Shaffer, R., Somers, M., \& Mehrotra, R. (2013). Medication reconciliation and therapy management in dialysis-dependent patients: need for a systematic approach. Clinical Journal of the American Society of Nephrology,8(11), 1988-
1999.

8. Pirmohamed, M. (2010). Drug-drug interactions and adverse drug reactions: separating the wheat from the chaff. Wiener klinische Wochenschrift, 122(3), 62-64.

9. Robertson, S., Penzak, S., Daniels, C. E., \& Dedrick, R. L. (2007). Drug interactions. In: Atkinson, A. J., Abernethy, D. R., Daniels, C. E., Dedrick R. L., \& Markey, S. P. Principles of clinical pharmacology, 229-247.

10. Courtman, B. J., \& Stallings, S. B. (1995). Characterization of drug-related problems in elderly patients on admission to a medical ward. The Canadian journal of hospital pharmacy, 48(3):161-166.

11. Krähenbühl-Melcher, A., Schlienger, R., Lampert, M., Haschke, M., Drewe, J., \& Krähenbühl, S. (2007). Drug-related problems in hospitals. Drug safety, 30(5), 379-407.

12 Rifkin, D. E., \& Winkelmayer, W. C. (2010). Medication issues in older individuals with CKD. Advances in chronic kidney disease, 17(4), 320-328.

13. Cardone, K. E., Bacchus, S., Assimon, M. M., Pai, A. B., \& Manley, H. J. (2010). Medication-related problems in CKD. Advances in chronic kidney disease, 17(5), 404-412.

14. Inagaki, H., Kuroda, M., Watanabe, S., \& Hamazaki, T. (2001). Changes in major blood components after adopting the supine position during haemodialysis. Nephrology Dialysis Transplantation, 16(4), 798-802.

15. López, M. P., Saliente, M. T. A., Monsalve, A. G., Cueva, M. A., Domingo, E. A., Hernández, M.,Carrion,C.C.,Marti,MM.,.C.,Querejeta,N.B.,Bla sco,J.B.,\& Milá, A. R. (2010). Drug-related problems at discharge: results on the Spanish pharmacy discharge programme CONSULTENOS. International Journal of Pharmacy Practice, 18(5), 297-304.

16. Vinks, T. H., De Koning, F. H., de Lange, T. M., \& Egberts, T. C. (2006). Identification of potential drug-related problems in the elderly: the role of the community pharmacist. Pharmacy World and Science, 28(1), 33-38. 